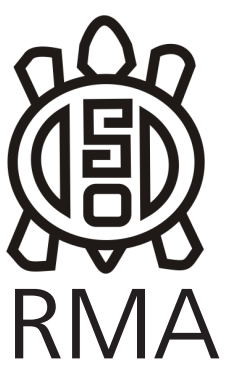

Arqueología

\title{
Conocimiento en relación. Reflexiones sobre el trabajo de campo arqueológico en el curso medio e inferior del Río Salado bonaerense
}

\author{
Virginia M. Salerno* y María Isabel González** \\ * CONICET, Instituto de Arqueología, Facultad de Filosofía y Letras, Universidad de \\ Buenos Aires, Argentina. vmasalerno@gmail.com \\ ** Instituto de Arqueología, Facultad de Filosofía y Letras, Universidad de Buenos \\ Aires, Argentina. igonzale@filo.uba.ar
}

\begin{abstract}
Resumen
En este artículo se reflexiona sobre el trabajo de campo, entendido como un momento clave del proceso de investigación arqueológica. Su importancia reside no sólo en que permite obtener materiales arqueológicos sino en que es una experiencia de creación de vínculos institucionales y personales. Además es una instancia de aproximación en/con el paisaje. El objetivo de este artículo es discutir el modo en que las relaciones sociales desplegadas durante el trabajo de campo (entre agentes, materiales, paisaje y conocimiento) impactan en la manera en que diversos agentes de la comunidad se vinculan con los arqueólogos, los materiales y el conocimiento que se busca generar. Con este fin se analiza con una perspectiva diacrónica, la experiencia de un equipo de trabajo de amplia trayectoria en la arqueología de la región pampeana bonaerense que viene realizando sus investigaciones en el curso inferior del río Salado, en particular en la localidad de Chascomús.
\end{abstract}

Palabras Clave: trabajo de campo arqueológico, arqueología y comunidad, historia de la arqueología pampeana.

Knowledge in relation. Thoughts on archaeological fieldwork in the middle and lower basin of the Salado river, province of Buenos Aires

\begin{abstract}
Fieldwork, conceived as a key moment in the process of archaeological research, is reflected on. In addition to its role in the recovery of archaeological materials, it is important as an experience of creation of institutional and personal relationships, and reconnaissance of and approach to the landscape and its inhabitants. The aim of this paper is to discuss the way in which social relations made during fieldwork have an impact on the way in which different agents within the community connect with the archaeologists, the materials, and the knowledge that is being generated. To this end, the experience of a work team with an extensive career in the archaeology of the Pampean region is analyzed from a diachronic perspective, namely the archaeological research carried out in the middle and lower basin of the Salado River, in particular in the district of Chascomús.
\end{abstract}

Keywords: archaeological fieldwork, archaeology and community, history of Pampean archaeology.

"Los días de trabajo de campo, los arqueólogos no sólo están ocupados con cuestiones tales como ¿Cuántos años tiene la unidad geológica? ¿Cuál es la procedencia de esta punta? sino también con asuntos mundanos y hasta triviales como ¿Cuándo regresará el cocinero del pueblo? ¿Qué diablos quieres decir con que tu destrozaste la puerta de la camioneta?"

D. H. Thomas, 1979: 146

Dentro de un proyecto de investigación arqueológica, el trabajo de campo se define y organiza como parte del conjunto de estrategias diseñadas para responder preguntas específicas, resolver un problema concreto o contrastar determinadas hipótesis (Carandini 1997, Renfrew y Bahn 2011). En esta instancia intervienen un grupo de especialistas y colaboradores en una serie de actividades destinadas a obtener materiales arqueológicos "in situ" y registrar esa evidencia. Si bien las tareas en el campo se organizan de acuerdo con procedimientos y reglas de razonamiento científico, constituidas históricamente, en su desarrollo se despliegan otro tipo de procesos relacionales que favorecen la creación de diferentes criterios valorativos. Como señala Thomas 
(1979), el trabajo de campo también incluye aspectos mundanos de la vida cotidiana: qué y cuándo comer, el cuidado del instrumental de campo, los momentos de descanso, entre otros. Nos preguntamos sobre el impacto de estos aspectos en las relaciones que los agentes locales establecen con los arqueólogos, la materialidad arqueológica y el conocimiento que se busca generar.

Para discutir estos aspectos, se presenta el caso de las investigaciones arqueológicas desarrolladas en la localidad de Chascomús, provincia de Buenos Aires. Con la elección de este caso, nos proponemos generar un acercamiento diacrónico y pormenorizado sobre la experiencia de un equipo de trabajo cuyas investigaciones comenzaron en el año 1985 y continúan en la actualidad. Esta continuidad posibilitó que se establecieran diversos lazos de colaboración, tanto personales como institucionales, que mediaron la producción y la gestión del conocimiento arqueológico del área. Si bien, el caso de Chascomús nos permite poner en perspectiva histórica los encuentros y desencuentros generados en el trabajo de campo, el análisis propuesto en este artículo se nutre de una experiencia de trabajo que no se limita a esa localidad. Las investigaciones arqueológicas que desarrolla nuestro equipo comprenden los municipios de Lobos, San Miguel del Monte, Lezama, Ranchos y General Belgrano, ya que articulan diferentes niveles de un problema de investigación referido al uso del espacio de humedales por parte de poblaciones cazadoras, recolectoras y pescadoras, en el curso inferior del río Salado. Es así como, el trabajo de campo en este proyecto de investigación se prolonga en el tiempo y se desarrolla en diferentes lugares.

El caso que aquí presentamos también es significativo para la comprensión del desarrollo de los estudios arqueológicos de las últimas décadas, en la región pampeana. A lo largo del tiempo, el equipo de investigación dirigido por una de las autoras (M. I. G.) estudió el modo de vida de poblaciones de cazadores recolectores que habitaron la zona en tiempos prehispánicos. Las preguntas y modos de abordaje utilizados se fueron organizando y legitimando en diálogo con otros arqueólogos que estaban trabajando en la provincia de Buenos Aires. En conjunto, estas propuestas fueron delineando agendas de problemas relevantes para investigar, modelos interpretativos y formas de aproximación cuya consolidación se manifestó en la organización de Jornadas Arqueológicas Regionales. La primera en Pigüé (1989) y la segunda en Chascomús (1991). En esos momentos se organizaron una serie de prospecciones, relevamiento de colecciones y/o museos, visitas a sitios arqueológicos y yacimientos paleontológicos de catorce localidades de la provincia de Buenos Aires, entre ellas Chascomús. En concordancia con los marcos interpretativos de la nueva arqueología, el trabajo de campo adquirió un lugar central y fue objeto de diversas discusiones que se desarrollan en la próxima sección.
Paralelamente, se realizaron jornadas de capacitación docente difundiendo los resultados de las investigaciones en curso (Bayón et al. 1991). La consolidación de estas investigaciones se reflejó en los Congresos de Arqueología de la Región Pampeana Argentina (CARPA) iniciados en el año 1998. En la actualidad ya se han celebraron seis de estos congresos.

Para este estudio hemos recurrido a diferentes vías de indagación que incluyen el relevamiento de informes y libretas de campo confeccionadas por el equipo de arqueología a lo largo del tiempo (entre 1985 y 2010), registro de observaciones en reuniones y trabajos de campo y entrevistas con agentes que han participado en esas actividades. El uso de estos múltiples registros nos permitió generar una perspectiva histórica y relacional. Los registros de observaciones y las entrevistas se realizaron en el marco de la investigación doctoral de una de las autoras, durante los años 2008, 2009 y 2010 (Salerno 2011-2012). En ese momento, el objetivo fue incorporar la visión de los agentes con respecto a sus experiencias y actividades según su pertenencia dentro de grupos sociales y/o espacios de interacción en los que, de distinta manera, la arqueología es parte. Para este artículo, la información proveniente de las entrevistas aportó una mirada retrospectiva sobre las actividades de campo, en la que los entrevistados elaboraron narrativas considerando su participación en eventos concretos. Por su parte, las observaciones, informes y libretas de campo nos permitieron poner en tensión diferentes lecturas de las entrevistas y los cuadernos de campo. Los informes y libretas se analizaron teniendo en cuenta que son una selección del investigador, con el propósito de registrar sus propios actos. Estos incluyen aspectos relacionados con la investigación en sí misma junto con factores técnicos, logísticos y éticos (Dabezies 2011). Es así que estos documentos pueden pensarse como productos de actos interpretativos que evocan representaciones de acciones en un momento concreto y en función de lo que se considera importante para la investigación en ese momento.

En la primera parte de este artículo se consideran diferentes aspectos que fueron discutidos por los arqueólogos en torno al trabajo de campo. A partir de esos aspectos, es posible rastrear las dimensiones "más representativas" que lo definen. Sin embargo, ellas no dan cuenta de la complejidad de las experiencias que ocurren durante el "trabajo de campo". En la segunda parte, discutimos esa complejidad a partir del análisis de nuestro caso de estudio. Proponemos que la experiencia del trabajo de campo permite la formación de vínculos entre los distintos agentes involucrados, los procesos que intervienen en la investigación y los diversos paisajes donde se trabaja. Paisajes que se convierten en un lugar simbólico, asociado a nuestras preguntas de investigación y al entusiasmo que nos impulsa a resolverlas. 


\section{El trabajo de campo en arqueología}

Muchos han sido los arqueólogos que se han propuesto como tema de discusión el trabajo de campo en Arqueología. Ya sea con fines didácticos y/o con el objetivo de generar una discusión teórico-metodológica de esta actividad, en este tipo de publicaciones encontramos explicaciones que remiten al trabajo de campo como una acción que implica un movimiento/viaje del investigador fuera de su espacio de trabajo y lugar de residencia, con el fin de ubicar y obtener materiales arqueológicos (Binford 1965, Gamble 2002, Hodder 1999, Refrew y Bahn 2011, Thomas 1979, entre otros). A lo largo del tiempo, en estos acercamientos encontramos diferentes fundamentos del trabajo de campo que remiten a diferencias en las formas de entender la arqueología, según contextos sociales, históricos e institucionales particulares (Guber et al. 2007). No es el objetivo de este espacio desarrollar una discusión sobre estos diversos fundamentos. Aquí nos proponemos indagar de qué modo estos aportes permiten articular ciertas concepciones comunes que nos permitan trazar una respuesta a la pregunta: ¿de qué hablamos cuando nos referimos al trabajo de campo?

Desde un enfoque de la historia interna disciplinar, varios autores indagaron sobre los modos en que los procedimientos y técnicas empleados durante el trabajo de campo, permitieron aumentar la integridad y validez de las colecciones arqueológicas, contribuyendo al "progreso" de la arqueología. Este es el caso de los estudios de síntesis y/o revisiones que abordaron la historia de la arqueología en Argentina (Boschin 19911992, Fernández 1982, Orquera 1987, Politis 1992, entre otros). A pesar de sus diferencias, estos autores proporcionan una macro-narrativa de la historia disciplinar signada por la sucesión de marcos teóricos que se "superan" a si mismos y que incluyen un "progreso" en el uso de técnicas, especialmente vinculadas con el trabajo de campo (centradas en problemáticas cronológicas y contextuales). Siguiendo estos estudios, puede decirse que durante las últimas décadas del siglo $\mathrm{XX}$ se generó un cambio fundamental en la Arqueología argentina, producto de la progresiva incorporación de métodos y recursos conceptuales promovidos por el movimiento estadounidense de la Nueva Arqueología (Binford 1965). Lejos de conformar un programa homogéneo y cerrado, la Nueva Arqueología representó un movimiento dinámico que se reformuló a lo largo del tiempo con el nombre de Arqueología Procesual (Johnson 2000).

La Nueva Arqueología generó un cambio conceptual en el modo de entender la arqueología y su objeto de estudio. En este replanteo, las nuevas formas de abordar y realizar el trabajo de campo tuvieron un papel primordial. Al respecto, Guber et al. (2007) señalan que en la Arqueología argentina, el cambio de perspectiva en relación con el trabajo de campo no sólo incluyó la incorporación de nuevas técnicas y el uso de instrumental específico, sino que implicó un cambio conceptual en la forma de articulación de esta instancia dentro de un proyecto de investigación arqueológica. Además, la inclusión de estudiantes en formación, le otorgó al trabajo de campo una función educativa esencial en la preparación de futuros investigadores (Guber et al. 2007). La importancia de las aproximaciones propiciadas por la Nueva Arqueología residió en que permitieron conectar modelos explicativos, generar resultados y promover nuevas discusiones en concordancia con los estándares científicos de esos momentos. Con el fin de estudiar la variabilidad cultural se diseñaron metodologías y teorías específicas del campo arqueológico como la teoría de rango medio, definiendo el "registro arqueológico" como objeto de investigación (Binford 1983). Esta concepción se diferencia de los abordajes previos que consideraban al pasado como el objeto a estudiar.

En la Arqueología argentina, estos marcos conceptuales se presentaron como una alternativa a las interpretaciones de la escuela Histórico Cultural. Su inclusión generalizada posibilitó la elaboración de modelos y estudios específicos sobre los materiales recuperados, promovió el uso de enfoques interdisciplinarios y el mejoramiento de las técnicas utilizadas para el registro y la conservación durante el trabajo de campo (Bayón y Flegenheimer 2003, Berón y Politis 1997, Boschin 1991-1992, Ceruti y González 2007, Fernández 1982, Gallardo y Cornejo 1985, Orquera 1987, Politis 1992, Politis y Madrid 2004, entre otros). Además, el enfoque procesual promovió el estudio de áreas y temas que habían sido escasamente considerados hasta el momento, entre ellos se destacan nuevas preguntas en el estudio de los grupos cazadoresrecolectores que habitaron la región pampeana (Mena Larraín 1989, Guber et al. 2007). Estos grupos dejaron de verse como una entidad homogénea y se enfatizaron los cambios diacrónicos. En el caso de las investigaciones en la localidad de Chascomús, estos enfoques permitieron construir nuevas miradas sobre el objeto, remarcando las peculiaridades areales que incluyeron aspectos vinculados con la actividad de pesca (C-R-P) y sus estrategias de movilidad (González 1997a, 1997b).

Estas macro-historias disciplinares resultan funcionales para promocionar la disciplina y gestionar sus recursos porque organizan el devenir disciplinar de acuerdo con conceptos cuyo valor es parte del presente en que se enuncian (Hurtado de Mendoza 2010). En contrapartida, invisibilizan la complejidad de relaciones sociales que caracterizan a la producción de conocimiento y que requieren un abordaje de la historia disciplinar, centrado en prácticas situadas, acotadas a momentos y lugares específicos. Al respecto se ha llamado la atención sobre los diversos modos en que los recortes regionales y locales en los que se desarrolla la Arqueología se articulan con el contexto mayor configurando situaciones particulares que no se explican mediante las narrativas generales 
(Endere 2005, Fontán 2005, Haber 2004, Mazzanti 2010, Podgorny 2004, Ramundo 2009, entre otros). En relación con el trabajo de campo, se destacó que uno de los efectos de la incorporación del procesualismo, fue la progresiva pérdida de valor cognitivo de conjuntos arqueológicos originados por coleccionistas y aficionados en comparación con las colecciones obtenidas mediante excavaciones sistemáticas (Pérez de Micou 1998). Además, otros estudios mostraron que estos cambios en las formas de valoración de las colecciones arqueológicas tuvieron lugar en el marco de procesos de profesionalización de la disciplina. También, como parte de este proceso se limitó la intervención de diversos agentes no especialistas (aficionados, estudiosos de los ámbitos locales y coleccionistas) en la construcción y validación de conocimiento sobre la materialidad arqueológica (Bonomo 2002, Pupio 2005, Salerno y Vigna 2012, entre otros).

En concordancia con estas perspectivas, varios autores señalaron el lugar central del trabajo de campo arqueológico en los procesos de distinción entre arqueólogos y otros agentes no especialistas. Esta diferenciación se entiende como una construcción social e histórica que prescribe formas de participación en relación con el conocimiento y sus referentes materiales tanto en los espacios del "campo" como en el "gabinete" (Bourdieu 2003, Latour 1990). Durante el trabajo de campo se elaboran criterios que se basan en el conocimiento del especialista en torno a las técnicas y dispositivos de registro empleados (Trigger 1992, Podgorny 2008, 2009). En estos procesos, la distinción de la actividad del arqueólogo no solo se define en relación con otros no especialistas (coleccionistas, buscadores de tesoros, pobladores locales, etc.), sino también en relación con otros especialistas que realizan trabajo de campo como por ejemplo, geólogos, paleontólogos y antropólogos (Guber et al. 2007, Nacuzzi 2002, Podgorny 2008). Los autores citados, destacan la complejidad de estos procesos de diferenciación, cuyas características específicas se definieron de acuerdo con los diversos contextos en los que se desenvolvió la práctica arqueológica. En provincia de Buenos Aires, el estudio planteado por Pupio (2012), advierte que estos sistemas de distinción no operaron de forma excluyente hasta mediados del siglo XX.

Otro tipo de discusiones en relación con el trabajo de campo han buscado aportar una mirada reflexiva sobre el mismo, destacando el valor cognitivo de esta experiencia. Estos argumentos resaltan que la actividad en el campo no es una instancia separada con respecto al momento de estudio de los materiales excavados (Amuedo y Kergarabat 2008, Hodder 1999). En tanto las interpretaciones y supuestos derivados de la experiencia del investigador en el campo permiten incluir el entorno de los hallazgos como parte de la "contextualización" del conocimiento generado (Bradley 2003, Hodder 1999). En la misma sintonía, algunos estudios valoran la recuperación del conocimiento práctico de los agentes locales en relación con el paisaje y con los materiales arqueológicos, llegando a replantear el modo en que se establecen las relaciones entre arqueólogos y estos agentes (Acuto et al. 2008, Delfino 2003, Endere y Curtoni 2006, Haber et. al. 2007). Lo interesante de estas discusiones, es que amplían la mirada con respecto a lo que entendemos por trabajo de campo y nos recuerdan que el proceso de la recolección y registro de materiales es una instancia de producción de conocimiento, donde los materiales ya comienzan a ser interpretados.

Finalmente, un aspecto que ha sido observado en relación con el trabajo de campo es su alto impacto y visibilidad pública en representaciones mediáticas y literarias que circulan ampliamente en la sociedad occidental (Conforti y Endere 2012, Hall 2004, Ramundo 2009, Refrew y Bahn 2011, Salerno 2009, Salerno y Pupio 2009). Excavación y hallazgos son dos caras altamente visibles del proceso de investigación arqueológica en detrimento de otras instancias/aspectos del mismo. En estas representaciones la arqueología es asociada con la aventura y el misterio (Lemaitre y Schall 2009). Además, resulta llamativo que la experiencia del trabajo de campo como parte habitual en la vida profesional del arqueólogo suele ser uno de los principales objetos de reflexión y en ocasiones es mencionado como un aspecto definitorio de la elección profesional. Los arqueólogos suelen mencionar como parte de las experiencias significativas de trabajo de campo el contacto directo con la naturaleza, la acción de desenterrar objetos y las relaciones que se establecen con los habitantes del lugar (Bianchi et al. 2008, CGA 2011, Matera et al. 2008, Rex González 2000). En estas representaciones el trabajo de campo se define a partir de la experiencia del viaje, el traslado de los miembros del equipo de investigación y la exploración del paisaje. Estos aspectos convierten al mismo en una experiencia exótica en términos noticiables, recordable en términos biográficos, irreemplazable para la producción de conocimiento.

En conjunto, puede decirse que las diferentes discusiones que los arqueólogos elaboraron en torno al trabajo de campo arqueológico se articulan en función de las prácticas involucradas y del desarrollo de técnicas y procedimientos destinados a la ubicación y obtención de materiales. Estas prácticas son consideradas centrales desde un punto de vista histórico, desde un punto de vista conceptual e inclusive en la representación pública de la arqueología. En los enfoques reflexivos, se incorpora a esta mirada la mediación que conlleva el vínculo con agentes locales. En todos los casos la centralidad otorgada a la obtención y la ubicación de materiales, nos remite a lo que el sociólogo Bernard Lahire (2006) llamó "polo más representativo de un domino de prácticas":

"en cada dominio de prácticas existe siempre un polo más "representativo" de ese dominio que los otros, un polo 
en el que se piensa en forma más espontánea cuando se evoca el dominio y que por eso mismo impide ver el resto del dominio" (Lahire 2006: 149).

En este trabajo nos preguntamos sobre esos otros aspectos que forman parte de lo no visible, lo no representativo del trabajo de campo y que, por esos motivos, su revisión requiere un ejercicio reflexivo (Bourdieu 2003). Para indagar estos aspectos en el próximo apartado presentamos el caso de Chascomús.

\section{Investigaciones arqueológicas en Chascomús}

En 1985 el Instituto de Ciencias Antropológicas de la Facultad de Filosofía y Letras de la Universidad de Buenos Aires (UBA) y el Museo Pampeano de Chascomús firmaron un convenio para formalizar la colaboración de un profesional en el ordenamiento de las colecciones arqueológicas del museo. Con esta mediación institucional y la obtención de una beca de iniciación otorgada por la UBA ${ }^{1}$, una de las autoras (M. I. G.) inició los primeros estudios arqueológicos sistemáticos en la localidad de Chascomús, donde eran muy escasos los trabajos previos. En ese momento el país se encontraba en un proceso de reorganización a nivel político e institucional producto de la apertura democrática. Los ámbitos universitarios en los que se desarrollaba la ciencia y dentro de ellos la Arqueología, se vieron favorecidos por la apertura de programas de investigación y de colaboración entre instituciones nacionales e internacionales, la formación de equipos de trabajo y el aumento de investigadores profesionales y becarios (Hurtado de Mendoza 2010, Politis 1992, entre otros). Particularmente, la UBA a través de su Secretaría de Ciencia y Técnica, inició un programa de becas de investigación promoviendo la institucionalización y jerarquización de esta actividad (Primeras Jornadas de Becarios de Investigación 1988). En consonancia, el Instituto de Ciencias Antropológicas de la UBA se reorganizó en diferentes secciones, con espacios concretos para el desarrollo de distintas líneas de especialización disciplinar: Antropología Biológica, Arqueología, Etnología y Folklore y a partir de 1985, Etnohistoria y Antropología Social (CGA 1989). Los trabajos de M. I. G. se desarrollaron en el marco de la Sección Arqueología².

En principio M.I.G. reclasificó las colecciones arqueológicas que estaban en el Museo Pampeano de acuerdo con nuevas categorías y preguntas generadas desde el marco teórico procesual. Uno de los principales objetivos

\footnotetext{
1 Beca interna de iniciación. Res. 1817/85. 1/3/86 al 30/5/88. Continuando con la Beca de Perfeccionamiento 1/6/88 al 30/5/91 luego Incorporada al Programa de Incorporación a la Docencia e Investigación de Becarios de Perfeccionamiento Res. (C.S.) n³258/88. 1991-1996 Secretaria de Ciencia y Técnica. Instituto de Ciencias Antropológicas. Facultad de Filosofía y Letras. Universidad de Buenos Aires.

${ }^{2}$ La Dirección del Instituto de Antropología estaba a cargo de la Dra. A. M. Lorandi y la Sección Arqueología a cargo de A. M. Aguerre.
}

de las investigaciones arqueológicas formulados, que se sostuvo a lo largo del tiempo, se relacionó con la posibilidad de construir conocimiento sobre los usos del espacio por parte de las poblaciones prehispánicas. Para su cumplimiento fue necesario indagar sobre la organización del paisaje, las características del ambiente natural, los procesos de transformación en escala regional y la ubicación de lugares con concentración de material arqueológico (González de Bonaveri 1989). En el diseño de estas investigaciones el trabajo de campo siempre tuvo un papel primordial. De acuerdo con los registros de las libretas de campo es posible afirmar que entre 1985 y 2010 el trabajo arqueológico incluyó por lo menos una salida al campo por año. En la tabla 1 se detalla la cantidad de salidas a lo largo del tiempo, como puede verse los primeros ocho años de investigación en el área estas actividades tienen una frecuencia mayor, llegando a un máximo de cinco campañas anuales en 1988. Durante estos primeros momentos de investigación, se priorizó el trabajo de campo debido a la ausencia de excavaciones arqueológicas previas en la zona y la necesidad de obtener materiales arqueológicos mediante la aplicación de procedimientos específicos. Si bien las colecciones existentes en el museo permitían generar algunos estudios, la arqueóloga sostuvo que "en realidad es la excavación arqueológica la que nos brinda una gran cantidad de información para interpretar procesos de devenir histórico" (González de Bonaveri 1988). Fue así como el equipo de M.I.G. realizó prospecciones, sondeos y excavaciones en puntos especificos del paisaje, vinculados con cursos de agua fluviales y lagunares, zonas no inundables y presencia de montes de tala (González de Bonaveri 1989,1991).

Con el paso del tiempo, la organización del trabajo de campo fue cambiando de acuerdo con las redefiniciones de la estrategia general del proyecto tales como: la formulación de nuevos problemas y preguntas de investigación, la disponibilidad fluctuante de recursos (económicos y humanos), las historias de vida de los miembros del equipo, la consolidación del equipo de trabajo, la incorporación de nuevos miembros, entre otros. El trabajo de campo se organizó promoviendo un enfoque interdisciplinario al incorporarse en el equipo el Dr. Marcelo Zárate, géologo especialista en el Cuaternario, con quien a través de la geoarqueología, se pudieron descifrar reconstrucciones geomorfólogicas y procesos de formación de sitios (González de Bonaveri y Zárate 1993/94, Zárate 1994).

Las diferentes actividades realizadas durante los trabajos de campo definieron en parte la duración (que varió entre tres a quince días) y la cantidad de miembros del equipo comprometidos en las salidas de campo (entre 2 y 8 personas muchas veces 16 al desdoblar los momentos de regreso y de llegada de un nuevo contingente en la misma temporada, quienes dirigían el trabajo permanecían a lo largo de ese tiempo). 


\begin{tabular}{cc}
\hline Año & Cantidad de trabajos de campo \\
\hline 1985 & 1 \\
1986 & 4 \\
1987 & 3 \\
1988 & 5 \\
1989 & 1 \\
1990 & 1 \\
1991 & 2 \\
1992 & 2 \\
1993 & 3 \\
1994 & 1 \\
1995 & 2 \\
1996 & 2 \\
1997 & 0 \\
1998 & 0 \\
1999 & 1 \\
2000 & 0 \\
2001 & 0 \\
2002 & 1 \\
2003 & 1 \\
2004 & 3 \\
2005 & 3 \\
2006 & 2 \\
2007 & 2 \\
2008 & 2 \\
2009 & 1 \\
\hline
\end{tabular}

Tabla 1. Cantidad de trabajos de campo realizados por el equipo de arqueología a lo largo del tiempo.

Table 1. Amount of field work carried out by the team of archeology over time

Además del trabajo de campo (realizado en ámbitos rurales), la organización del trabajo arqueológico incluyó diferentes actividades que pueden diferenciarse de acuerdo con los contextos sociales e institucionales involucrados en las mismas. Se incluyen las gestiones e indagaciones en instituciones locales (archivos, museos, otros) y las acciones destinadas a la socialización del conocimiento en ámbitos académicos (congresos, universidades), y no académicos (con medios masivos de comunicación, museo, escuelas). Cada una de estas instancias reunió momentos y actividades específicas cuyos objetivos se enmarcaron dentro de la estrategia mayor del proyecto. Todas ellas, son actividades superpuestas en un continuo temporal que en este caso suma 25 años de trabajo sistemático. Aquí nos abocaremos a considerar algunos aspectos referidos al trabajo de campo y al modo en que las relaciones, experiencias y saberes que se ponen en juego durante esta actividad, posibilitaron que se generaran espacios de discusión y resignificación de los materiales y de los conocimientos arqueológicos. Con este propósito, a continuación analizamos el trabajo de campo desde la óptica de las relaciones en las que se desarrolló esta actividad, teniendo en cuenta el papel de lo institucional, las visitas y las interacciones cotidianas durante esta actividad.

\section{Un espacio de mediación institucional}

"En Chascomús nos recibe $\mathrm{HB}$, quien nos traslada al museo. Allí conversamos sobre efectuar un trabajo a nivel divulgación para publicar. Llegamos a la Intendencia hablo con $X$ quien me comunica con $\mathrm{R}$ para arreglar viaje al río Salado. El Director PP me consulta sobre antigüedad de unas valvas halladas en la cordillera. Somos recibidas por el Intendente, quien se interesó por nuestro trabajo y prometió ayuda de vehículos, autorizándonos a viajar con los chicos al Salado [se refiere a los transportes escolares que el municipio dispone para los chicos que viven en áreas rurales sin escuela cercana]. Nos traslada el Sr. P a lo de PP y allí instalamos la carpa. Caminamos para llegar al casco de la horqueta..." (Registro en libreta de campo, 20 de febrero de 1987).

"Por la mañana nos vino a buscar un camión de la municipalidad, llegamos al museo 11:30 de la mañana, separamos el material que queda en el museo y lo que regresa a Buenos Aires [sigue detalle del inventario] $A B$ hizo que una combi de la municipalidad nos llevara hasta Buenos Aires con todo el equipaje. Conversamos acerca de los resultados de la excavación" (Registro en libreta de campo, 15 de marzo de 2003).

Las citas con las que iniciamos este apartado refieren a un momento particular, sistemáticamente registrado en las libretas de campo: los inicios y finales de una temporada de trabajo. La particularidad de estos momentos reside en que se involucran diversos lugares, agentes e instituciones locales. Se trata de movimientos en la ciudad, donde el museo municipal de la localidad ${ }^{3}$ participó como institución mediadora, lugar de "posta": es a donde el equipo llega desde Buenos Aires (a veces incluyendo estudiantes que arribaban de la Plata y/o Bahía Blanca), se reorganiza y continúa viaje al campo y a la inversa cuando se vuelve del campo. Es un momento fugaz, los arqueólogos están ansiando estar en otro lado (o llegar al campo o regresar a sus casas luego del trabajo de campo), los agentes del museo interrumpen su quehacer cotidiano, reciben y colaboran con la futura campaña, y/o se ponen al corriente de lo que sucedió en la campaña. Usualmente el equipo de arqueología deja cajas con herramientas de campo en la institución (cuyo inventario se detalla en las libretas de campo cada fin de campaña) y avisa de contactos nuevos, producto de los intercambios con pobladores locales durante la estadía en el campo, para que el museo pueda gestionar futuros permisos de acceso con los propietarios. En ocasiones, durante estos encuentros el museo invitó a periodistas locales que realizaron breves entrevistas, para difundir en

\footnotetext{
3 Museo Pampeano de la Municipalidad de Chascomús, en adelante se mencionará como museo
} 
los medios masivos locales el proyecto de investigación.

Durante los trabajos de campo, los investigadores estuvieron condicionados en su actuar por los permisos de ingreso a las propiedades rurales, así como por aspectos prácticos afines al movimiento e instalación de personas (estudiantes y profesionales) y recursos (desde carpas y bidones de agua hasta herramientas para el registro y conservación de materiales arqueológicos). El museo local tuvo un papel importante como facilitador para el acceso a los lugares y el establecimiento de los contactos iniciales con los dueños de los campos. Mientras que la organización logística fue variando de acuerdo con las colaboraciones recibidas no sólo por el museo sino también por diversos agentes locales. En todos los casos, el museo siempre ofreció su respaldo y garantía a los investigadores.

Anteriormente hemos mencionado el contexto nacional de transformaciones políticas, sociales e institucionales en que se iniciaron los trabajos de investigación arqueológica en esta zona. Para este trabajo, interesa destacar que en la localidad de Chascomús, estas transformaciones tuvieron su eco en reorganizaciones a nivel institucional. En el museo se reemplazaron los agentes a cargo, se incorporaron jóvenes profesionales en un programa de becarios del municipio y se formuló una agenda tendiente a modernizar y reciclar las exposiciones de las diferentes salas del museo. En este marco, el acompañar e impulsar un proyecto de investigación científica respaldado por la Universidad de Buenos Aires fue un elemento que contribuyó con el nuevo perfil que la institución estaba elaborando. Mientras que para el equipo de arqueología, el trabajo de campo fue/es una acción indispensable en tanto es el momento en que se "recuperan" los vestigios que sustentan las investigaciones. Para el museo local, los trabajos de campo fueron instancias que prometieron nuevos materiales para la exposición y nuevas posibles formas de participación y actuación institucional vinculadas con las actividades de difusión de los materiales y los conocimientos generados.

Así por ejemplo, durante los primeros diez años de investigación, la Asociación Amigos del museo tuvo un importante rol como difusor y soporte material de las actividades. Estas acciones posibilitaron el acercamiento de colaboradores locales con el equipo de arqueología, a la vez que contribuyeron a ampliar el prestigio del museo. En una entrevista a un miembro de la Asociación Amigos, éste recordó que colaboraban con los trabajos arqueológicos principalmente en la organización de eventos de difusión y en la logística del trabajo de campo:

"A raíz de mi incorporación al museo tuve la oportunidad de conocer a María Isabel, aunque yo había asistido a algunas de sus charlas. Ahí empezamos a conversar mucho y a veces ayudaba a preparar cosas que llevaban para realizar el trabajo de campo (en particular me acuerdo cuando iban a La Guillerma (creo así se llamaba el sitio arqueológico). Participaba GG. Conocí varios arqueólogos ya que participé de todas las reuniones que se hacían en el museo. Me acuerdo en especial una que venía una chica tipo de alemana, muy alta y con la característica de arqueóloga. Después a Isabel la vi dando charlas sobre lo hallado en sus trabajos en la sala del museo, donde recuerdo había pedazos de gliptodontes, piedras, restos indígenas. Era una sala más." (Entrevista ex integrante de la Asociación Amigos, 2 de agosto y 30 de julio 2010)

Un evento especialmente mencionado en estas memorias fue cuando:

"Conseguimos un subsidio4, usamos la mitad para hacer un fechado y la otra para arreglar el edificio. El fechado se hizo en Estados Unidos, nos sentíamos muy importantes, era algo que se hacía por primera vez, acá..." (Entrevista ex integrante de la Asociación Amigos, 15 de marzo del 2011)

La relación entre arqueólogos y museólogos se fue redefiniendo con el paso del tiempo. Algunos puntos estables fueron la sostenida colaboración entre las instituciones involucradas (Museo Pampeano Municipal y Universidad de Buenos Aires), la organización de actividades educativas en conjunto y las diversas formas de apoyo que el museo sostuvo para el trabajo de campo arqueológico. Entre las acciones que generaron alto impacto y visibilidad en la localidad, se destacan una serie de encuentros cuya sede fue el museo. Entre los años 1989 y 1995, se realizaron de forma anual, jornadas de Arqueología local en las que se difundieron los resultados de las investigaciones locales junto con arqueólogos, geólogos y paleontólogos invitados que presentaron una actualizacion de sus investigaciones en distintas regiones del país. Además, en el año 1990 se llevaron a cabo las Primeras Jornadas de Capacitación Docente y Participación Arqueológica y Antropológica en la provincia de Buenos Aires. Como resultado de estos encuentros se formó un banco de datos con alumnos de escuelas secundarias locales interesados en participar en las tareas de campo. A raíz de ello, algunos estudiantes de esas instituciones participaron como ayudantes de campo. De esta manera, se convirtieron en agentes multiplicadores en tanto sus experiencias fueron compartidas en sus escuelas mediante charlas con apoyatura visual elaboradas en conjunto con el equipo de investigación.

Desde el punto de vista institucional, es posible decir que el intercambio generado, conllevó la introducción de otros criterios para pensar, clasificar e interpretar las colecciones arqueológicas del museo. Criterios que se

\footnotetext{
${ }^{4}$ Se refiere a un subsidio para la investigación arqueológica otorgado a la Asociación Amigos del Museo Pampeano. Cámara de Diputados de la Nación Art. 34 Ley 24191 Orden n²832/94. Investigadora responsable: María Isabel González.
} 
vieron reflejados en el año 1992, en el reciclado de la sala "Arqueología e indígena" que pasó a llamarse "Sala Arqueológica-Paleontológica" (Salerno y Vigna 2012). En este proceso la continuidad y el lugar preponderante del trabajo arqueológico hicieron que la gestión de la materialidad prehispánica del museo fuera parte de un proyecto más amplio que excedió el rol educativo de la exhibición de las colecciones. Por ello en los criterios y formas de pensar la exhibición se incluyó todo el proceso de investigación arqueológica: recolección de datos, análisis y transferencia. En este caso, el enfoque reflejó la separación de la Paleontología y la Arqueología posibilitando la afirmación de esta última. Además se reformuló la historia de las poblaciones prehispánicas mediante el uso de categorías temporales elaboradas desde la Arqueología.

En el año 1995 se organizó una exposición temporal en el Archivo Histórico y Museo del Banco de la Provincia de Buenos Aires de la Capital Federal. Esta muestra no sólo sintetizó la información generada durante los primeros diez años de trabajo arqueológico en la zona, también expresó la consolidación del intercambio institucional entre el Museo Pampeano y la Universidad de Buenos Aires. De hecho, el diseño y el montaje expositivo se coordinó entre agentes del museo y del equipo de arqueología. En la muestra se expuso el trabajo de campo representado en una cuadrícula hecha con tierra, cordeles, cucharines, etc. Además, en la redacción de los guiones participaron otros arqueólogos que trabajan en la provincia de Buenos Aires incluyendo la información sobre las poblaciones prehispánicas de la localidad dentro de una narrativa mayor vinculada con los procesos de poblamiento de la región pampeana. Información que se reprodujo en los distintos medios locales. Además, a esta muestra asistieron las autoridades municipales y se organizaron la visita de contingentes escolares. En total hubo alrededor de mil personas que visitaron la exposición en el período que estuvo abierta.

Desde estas primeras exposiciones, han transcurrido veinte años. En este tiempo las distintas salas del museo fueron objeto de reestructuración y de diversas acciones destinadas a la conservación de las colecciones. En el caso de la sala arqueológica su actualización en el año 2012, se realizó como parte del intercambio entre arqueólogos y museólogos, como un paso necesario para incluir nueva información producida a través de las investigaciones en curso e incorporar los nuevos materiales de exposición al museo.

De la misma manera que se involucraron instituciones y agentes locales, las actividades de campo junto con los eventos de comunicación fueron ampliamente difundidas en los diarios locales (Salerno 2011-2012). En la representación mediática de los primeros años de trabajo de campo, las noticias sobre su realización, se acompañaron con agradecimientos por las buenas disposiciones de los dueños de los campos, la donación de agua por parte de una empresa local, siempre se destacó la participación de estudiantes de la localidad, entre otros. Tal como se planteó en otro trabajo, estas representaciones se focalizaron en el relato sobre el trabajo de campo (actividad realizada por los profesionales) más que en el contenido generado mediante estas actividades (el pasado investigado). A tal punto que el producto generado (conocimiento) se fusionó con el proceso de producción (la investigación arqueológica), haciendo que el primero se constituyera en un bien/valor exclusivo de los especialistas. Así la información arqueológica sobre el pasado local fue referida mediante imágenes y descripciones de la materialidad categorizada en términos de "hallazgos arqueológicos", actividades desarrolladas en el campo, instituciones y agentes (especialistas y colaboradores locales) que intervinieron en su construcción (Salerno 2008). Ahora bien, en este modo de hacer público el trabajo arqueológico, entendemos que la importancia otorgada a la participación de agentes locales, construyó una vía indirecta de inclusión/participación en la investigación arqueológica. Inclusión investida de prestigio en tanto la participación local se presenta como primordial para la realización de las actividades.

\section{Visitas y testigos en el campo}

"Nos entusiasmábamos, cada vez que ellos encontraban cosas... una vez trajeron unas piedritas y uno decía, ¿y esto para que les puede servir? para ellos eran valiosísimas (...), ellos hablaban todo el día, venían, tomaban mate, y contaban lo que encontraban. Al final uno sin entender viste, pero también se entusiasma y contentos de que ellos pudieran encontrar cosas. (...) era un hombre interesante [en referencia a un investigador acompañante], que se ponía a conversar, ahí en la mesa, te podía dar una lección hasta a uno que no sabía nada..." (Entrevista a poblador rural, 10 de marzo 2011)

"Se pudo observar en el curso de la citada visita, el alboroto producido, al creer haber encontrado un resto fósil, que resultó ser el borde de una tinaja colocada boca abajo, que al ser extraída mostraba una serie de incisiones prolijamente realizadas y un espesor en el tratamiento de la arcilla realmente encomiable" (Diario El Imparcial, 31 de marzo 1992)

Las visitas de diferentes agentes al lugar donde los arqueólogos excavaban resultaron experiencias significativas en diferentes sentidos. En principio, es de destacar que en este caso de estudio la presencia en el campo de agentes que no pertenecen al equipo de arqueología fue esporádica y ocurrió en el marco de diferentes tipos de visitas. Esto fue así porque las excavaciones se realizaron en lugares de difícil acceso, tanto por las distancias como por tratarse de propiedades privadas.

Por un lado, se destacan las visitas realizadas por los pobladores rurales que vivían en los campos cercanos. 
En las libretas de campo, se encuentran registros de explicaciones de agentes locales sobre el paisaje y sobre el modo de realizar determinadas actividades (sobre cómo X persona procesó un animal de caza, formas de preparación de alimentos, si en tal o cual lugar antes había o no un monte de tala). También figura información sobre las características de colecciones privadas con pocos objetos, así como referencias sobre lugares donde los habitantes del ámbito rural encontraron materiales. En algunos casos, se mencionan intercambios de bibliografía y préstamos de materiales, informaciones sobre ubicación de material y sobre cómo llegar a ciertos lugares. Además, en estos registros también aparecen datos de nuevos contactos con pobladores locales establecidos durante la estancia en el campo. Este intercambio de material e información tuvo lugar en el marco de relaciones informales que se establecieron con distintas personas. Así se destaca que los pobladores rurales suelen ayudar a los miembros del equipo de investigación para que éstos puedan instalarse. Se trata de una colaboración espontánea que es parte de la vida cotidiana, sin por ello dejar de ser primordial en el éxito de la actividad. Durante este tiempo, los arqueólogos conviven con personas que en ocasiones, se convirtieron en esporádicos ayudantes de campo. En estas relaciones se construyeron amistades y conocimientos mutuos, en tanto parte de las interacciones refieren al trabajo que se está haciendo: en relación con las características de los materiales hallados, sobre interpretaciones hipotéticas elaboradas en el campo, procedimientos metodológicos, etc.

Otro tipo de visitas, son las generadas por pobladores de la ciudad y/o funcionarios municipales que llegaron al sitio para conocer el trabajo. Si bien se trata de encuentros poco usuales, cuando ocurrieron, estos eventos fueron importantes para generar visibilidad y otro tipo de lecturas sobre el trabajo de campo. Encontramos información sobre estas visitas en las libretas de campo y en algunos casos, en publicaciones de los periódicos locales. Así por ejemplo, una de las citas que antecede este apartado relata parte de la experiencia de la Directora de Cultura municipal, quien visitó a los arqueólogos en el lugar de excavación en el año 1992. El relato de lo observado se publicó en varios periódicos locales con detalles sobre la forma de excavación y el tipo de materiales hallados. Mediante estos relatos se difundieron representaciones del trabajo arqueológico que se centran en el "esfuerzo" y la "paciencia" de los arqueólogos durante su día de trabajo, conformando alternativas a la tradicional mirada de los arqueólogos como "exóticos aventureros". Otro tipo de reflexiones realizadas por agentes que visitaron el lugar se refirieron a un componente energético derivado del estar en el campo, expresado en términos de fuerza y energía trasmitida en determinados puntos del paisaje.

También encontramos registrado en libretas de campo y en publicaciones mediáticas la presencia de docentes y estudiantes de la escuela rural cercana. En estos casos, se resaltó la experiencia de compartir el día de trabajo, el propósito educativo de los encuentros, las explicaciones proporcionadas por los arqueólogos sobre su trabajo. La movilización de los estudiantes en estas visitas se realizó con la participación de padres que colaboraron para los traslados con sus vehículos. En este tipo de encuentros se establecieron importantes lazos con otros pobladores rurales, que permitieron intercambiar conocimiento con relación a la localización de materiales arqueológicos en el paisaje.

Finalmente, otro tipo de visitas lo constituyen aquellas generadas por otros especialistas, colegas de los investigadores. Un evento especial estuvo dado por un recorrido organizado en el sitio arqueológico La Guillerma en el año 1993, como parte del trabajo de campo de un curso sobre Geoarqueología dictado por el Dr. Marcelo Zarate en la Universidad de La Plata. Esta actividad se realizó con el doble objetivo de generar una experiencia didáctica, así como de propiciar un acercamiento interdisciplinario para el estudio de la localidad arqueológica. La presencia de expertos, tanto arqueólogos como geólogos, favoreció la discusión en el terreno acerca de la formación de los sitios, de las características de los materiales y dentro de ellos particularmente los referidos a la cerámica. Fueron especialmente interesantes las puestas en común referidas a las geoformas de estos paisajes a partir de los cuales se pudo caracterizar a esta localidad con el concepto de "sitio somero". Esta categoría fue luego discutida en un trabajo específico (Zárate et al. 2000/2002). Además, algunos arqueólogos/as presentes, eran investigadores del NOA argentino con una vasta experiencia en el análisis de la cerámica de aquella región, lo que favoreció la discusión acerca de diferentes aspectos de la manufactura, acabado y decoración de los materiales cerámicos pampeanos.

Otros especialistas que participaron del trabajo de campo fueron ceramistas locales, quienes asistieron a las prospecciones y/o excavaciones en diferentes oportunidades. Las experimentaciones iniciales abrieron el camino para la formulación de un diseño de arqueología experimental tendiente a la replicación de materiales cerámicos bajo condiciones científicas cuidadosamente controladas. A partir de estos trabajos, la ceramista A. Francese comenzó a integrar el equipo de investigación arqueológica y se generaron réplicas de vasijas obtenidas en la cuenca del río Salado, para una exposición en el Museo y Archivo Histórico de Bahía Blanca realizada en el año $2003^{5}$ (Pupio et al. 2004). Estos intercambios posibilitaron la objetivación del conocimiento de la cerámica arqueológica en términos de contenido curricular del Instituto Superior de Formación Artística (ISFA) de la localidad (Salerno 2011-2012). Se abrieron así nuevos caminos de participación y acción en relación con la cerámica prehispánica. En el año 2011, un proyecto generado en el ISFA y subsidiado por el Ministerio de Educación, posibilitó que un grupo de ceramistas dirigidos por A. Francese, realizara un conjunto de réplicas

\footnotetext{
5 Museo y Archivo Histórico de Bahía Blanca. Inauguración Sala Permanente de Arqueología: 5 de diciembre de 2003. Proyecto coordinado por la Dra. A. Pupio. Municipalidad de Bahía Blanca.
} 


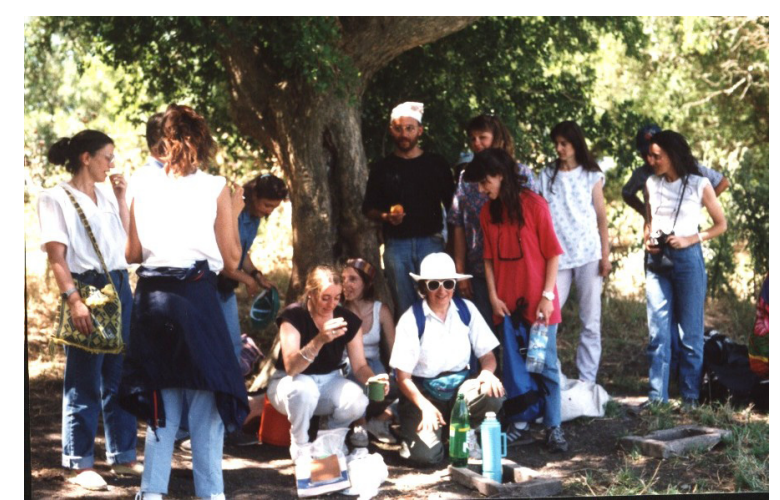

Figura 1. Visita al sitio arqueolgico La Guillerma, año 1993.

Figure 1. Visit to La Guillerma archaeological site, 1993.

cerámicas que actualmente se exponen en el museo local (Francese et al. 2011).

Para concluir este apartado, es de destacar que en todos los casos, las visitas de agentes que ocasionalmente llegaron al lugar, junto con los colaboradores en el campo y las visitas organizadas en el marco de actividades educativas y de intercambio con otros especialistas, permitieron hacer públicas diferentes miradas sobre la actividad del trabajo de campo. Los visitantes elaboraron relatos sobre lo que observaron, relatos que se organizaron en diálogo con la propuesta de los propios arqueólogos. Los mismos resaltaron y caracterizaron diferentes aspectos seleccionados por los visitantes que permitieron generar lecturas heterogéneas en torno a cómo se entiende la actividad del arqueólogo, el sentido de la investigación, la caracterización de los materiales hallados, entre otros. De este modo, en los intercambios cotidianos, se elaboraron y legitimaron diversos criterios a partir de los cuales se reivindicó el conocimiento en torno a los materiales arqueológicos. Por ejemplo, el trabajo de campo generó visibilidad y reconocimiento para los agentes locales, de pequeños fragmentos de cerámica, restos arqueofaunísticos, lascas y microlascas, como parte de lo "arqueológico". De la misma manera, para los arqueólogos fueron trascendentes los relatos sobre los usos del paisaje en momentos de secas e inundaciones, el empleo de recursos vegetales, las narraciones orales en torno a "vasijas de indios", la ubicación de hallazgos "ocasionales" al arar los terrenos (principalmente boleadoras de piedra). Estas experiencias relacionadas con los materiales arqueológicos refieren a un modo de "existir" de dichos materiales y junto con ellos, del pasado indígena que evocan. En el caso de las visitas de otros especialistas, debe agregarse que éstas contribuyeron a la legitimidad de la investigación que se estaba realizando. No sólo porque dieron cuenta del reconocimiento del trabajo del equipo de arqueología en el campo, también porque en esos intercambios se consensuaron formas de interpretación y análisis de los materiales y su paisaje.

\section{Palabras finales}

El punto de partida de este artículo fue abordar el trabajo de campo como un espacio relacional, que involucra agentes, materiales y paisaje. Con esta aproximación buscamos llamar la atención sobre diversas prácticas cotidianas que, siendo centrales al trabajo de campo arqueológico, raramente son objeto de reflexión por parte de los agentes involucrados (Bourdieu 2003). Por ello es lícito pensarlas como "micro prácticas" que median la producción de conocimiento durante el trabajo de campo. En este trabajo destacamos los intercambios cotidianos producidos en el marco de diversos tipos de visitas en el campo, así como la mediación institucional en la que se despliega esta instancia de la investigación. En conjunto, estos aspectos del trabajo de campo remiten a la dimensión relacional del proceso de construcción de conocimiento. Como práctica social, la organización de los saberes en objetos de conocimiento implica un recorte de la realidad, así como una selección de la forma de conocer y de comunicar lo conocido que remiten a diferentes posicionamientos y experiencias de los agentes participantes (Beillerot 1998). En nuestro caso de estudio, puede verse cómo a lo largo del tiempo, el trabajo de campo promovió diferentes tipos de acercamientos con conocimientos y materiales arqueológicos, a la vez que habilitó espacios públicos de discusión en torno a los mismos.

Así, como parte de la cotidianeidad del trabajo de campo, la participación de pobladores rurales y las diversas visitas descriptas, fueron instancias en que se elaboraron criterios de valoración sobre esta actividad, los materiales arqueológicos y los usos del espacio. Desde la arqueología se utiliza una noción inclusiva de lo que se entiende como arqueológico que se amplía de acuerdo con patrones construidos en el marco de la disciplina. Mientras que los agentes colaboradores y participantes esporádicos, dieron distinta importancia a criterios vinculados tanto con el tipo de material, tamaño y fragmentación de un objeto, como con características objetivadas en el paisaje, discusiones en relación con la toponimia, experiencias de vida en un determinado lugar y/o historias e imágenes populares trasmitidas de forma oral. En estos procesos, se destaca la importancia del conocimiento práctico de los agentes locales y el hecho de que, en ocasiones, los criterios a partir de los cuales se construyen valoraciones sobre los materiales, se articulan con representaciones estereotipadas producto de las experiencias y los modos en que se organizó el saber en torno a los pueblos originarios de la zona desde el poblamiento hispánico (Salerno 2011-2012).

También es importante recordar que gran parte de las visitas de este caso de estudio remiten a actividades educativas como parte de las actividades del trabajo de campo. Las mismas incluyeron visitas de estudiantes universitarios vinculados con la arqueología como 
estudiantes de escuelas secundarias y primarias de la zona. Los visitantes se convirtieron en testigos/ colaboradores de la acción cuyo papel fue importante para generar veracidad en torno a la misma. En este sentido, posibilitaron replicar y validar representaciones en relación con los materiales arqueológicos y el propio trabajo de campo.

De la misma manera, pensar el campo como espacio de mediación institucional, conlleva a adentrarnos con las relaciones de poder y saber que se desplegaron. Requiere reflexionar sobre la actividad del trabajo de campo como disparadora de prestigio, movilización de recursos y personas. Aspectos que para el caso de estudio no estuvieron exentos de conflictos y negociaciones entre los diferentes sujetos involucrados. El contexto institucional define parte de las limitaciones y posibilidades de la realización de la investigación en términos de tiempo, recursos materiales disponibles, diferentes exigencias y objetivos en relación con la investigación. En el caso presentado, la realización de los trabajos de campo se organizó en conjunto con el museo local, en tanto esta institución facilitó el acceso a los lugares, estableció los contactos iniciales con los dueños de los campos y ofreció su nombre como respaldo y garantía para los investigadores. Por su parte, los medios masivos difundieron representaciones sobre la actividad y el trabajo que se realizó, promoviendo otros espacios públicos de discusión de esta actividad. A su vez, estos intercambios propiciaron la inclusión de diferentes colaboradores locales con el equipo de arqueología. Con el transcurrir del tiempo se fue construyendo un sentido de pertenencia y participación por parte de los agentes locales en relación con la investigación arqueológica. Hecho que convirtió este trabajo de investigación en un tema de interés compartido por parte de las instituciones participantes, aunque con disímiles objetivos y necesidades. En el caso de los vínculos con el museo, las diferencias remiten a distintas expectativas y prácticas en torno a la materialidad arqueológica que es entendida en un caso, como objeto de exposición y en otro, como objeto de análisis. No se trata de concepciones contrapuestas sino de miradas que acentúan dos dimensiones diferentes de la materialidad e involucran distintas prácticas y tiempos. A su vez, en el marco de estas trayectorias institucionales se forjaron diversos vínculos entre los agentes del museo y los miembros del equipo de arqueología que incidieron de diferente manera en la gestión y producción de conocimiento sobre el pasado prehispánico en la localidad.

En fin, lejos de presentar reflexiones acabadas, consideramos que el interés principal de este artículo radica en llamar la atención sobre las diversas prácticas involucradas en el trabajo de campo y aportar al debate sobre la incidencia de las relaciones desplegadas durante esta instancia en los procesos de construcción y resignificación del conocimiento arqueológico. En el caso aquí presentado, la información arqueológica producida, en ocasiones se convirtió en herramienta-insumo para la realización de proyectos personales, principalmente artísticos, literarios y docentes (Francese et al. 2011, González de Bonaveri, M.I. y M.M. Frère 1998, Salerno 2011-2012). En otros casos y de forma discontinua, estas interacciones se convirtieron en acciones que sirvieron tanto a agentes del equipo de arqueología como a diversos agentes locales. Por ejemplo, se facilitaron diversos aspectos logísticos y circuló información sobre lugares con materiales arqueológicos. En conjunto, estos intercambios posibilitaron los reconocimientos recíprocos (entre arqueólogos y agentes e instituciones locales así como con otros especialistas) y la producción de sentidos en torno a la materialidad tanto para arqueólogos como para los distintos agentes que participaron en ellos. En estos movimientos, los criterios sobre lo que formó parte del material arqueológico se ampliaron y redefinieron, en ocasiones interpelando las categorías del propio investigador. Además, estos procesos pusieron de manifiesto el carácter colectivo de la construcción del conocimiento arqueológico.

Ciudad de Buenos Aires, 15 de abril de 2013

\section{Agradecimientos}

Las autoras agradecen la colaboración de la Lic. Cristina Bayón y la Dra. Ana María Presta. Al Dr. Marcelo Zárate y a todos los que participaron, a lo largo de los años, en los trabajos de campo. A los colegas que visitaron el sitio La Guillerma en el año 1993. Este trabajo fue realizado en el marco de los proyectos UBACyT 2011201420020100100134 y PICT 01517.

\section{Bibliografía}

Acuto, F., C. Amuedo, M. Kergaravat, A. Ferrari, L. Gamarra y A. L. Goldin. 2008. Experiencias subjetivas en las aldeas prehispánicas del valle Calchaquí Norte: Arqueología de la vida cotidiana, prácticas y relaciones sociales durante el Período Prehispánico Tardío. Borrero L.A. y N. Franco. Arqueología del extremo sur del continente americano. Resultados de nuevos proyectos, 11-54, CONICET e Instituto Multidisciplinario de Historia y Ciencias Humanas, Buenos Aires.

Amuedo, C. y M. Kergaravat. 2008. El cucharín por el mango: propuesta para la aplicación de métodos reflexivos en el trabajo de campo. La Zaranda de Ideas, 4: 147-152.

Bayón C. y N. Flegenheimer. 2003. Tendencias en el estudio del material lítico. Análisis, interpretación y gestión en Arqueología de Sudamérica, 65- 90.

Bayón, C. M. Berón, N. Franco, M. I. González, P. Lozano y F. Oliva. 1991. Algunas reflexiones sobre las primeras 
jornadas de participación arqueológica y antropológica en la provincia de Buenos Aires. Boletín del Centro, 3: 53-64.

Beillerot, J. 1998. Los saberes, sus concepciones y su naturaleza. Beillerot, J., C. Blanchard-Laville y N. Moscón, Saber y relación con el saber 19-42. Paidós, Buenos Aires.

Berón, M. y G. Politis. 1997. Arqueología Pampeana en la década de los '90. Estado de las investigaciones y perspectivas. Berón M. y G. G. Politis, Arqueología pampeana en la década de los '90, 7-32. Museo de Historia Natural /INCUAPA. San Rafael/ Olavarria

Bianchi, P. E., V. Salerno, C. Squitieri y M. Vigna. 2008. Una Charla con Ana Margarita (Annet) Aguerre, arqueóloga. Pupio, A., R. Frontini, M. M. Frère y N. Mazzia, Sobre los primeros pobladores de la pampa bonaerense. Apuntes de arqueología para llevar a la escuela, Departamento de Humanidades, UNS y F. F. y L. UBA, Buenos Aires.

Binford, L. 1965. Archaeological systematics and the studie of culture process. American Antiquity 30: 203210.

Binford, L. 1983 [1991]. En busca del pasado: descifrando el registro arqueológico. Critica, Barcelona.

Boletín del Centro. 1991. Publicación del Centro de Registro del Patrimonio Arqueológico y Paleontológico. 2

Boletín del Centro. 1992. Publicación del Centro de Registro del Patrimonio Arqueológico y Paleontológico. 3

Bonomo, M. 2002. El hombre fósil de Miramar. Intersecciones en Antropología 3: 69 - 87.

Boschín, M.T. 1991-1992. Historia de las investigaciones arqueológicas en pampa y patagonia. Runa, XX:111-114.

Bourdieu, P. 2003. El oficio del científico. Ciencia de la ciencia y reflexividad. Curso del College de France 2000 - 2001. Anagrama, Barcelona

Bradley, R. 2003. Seeing Things. Perception, experience and the constraints of excavation. Journal of Social Archaeology 3 (2):151-168.

Carandini, A. 1997. Historias en la tierra: manual de excavación arqueológica. Critica, Barcelona.

Ceruti, C. N. y M. I. González. 2007. Modos de vida vinculados con ambientes acuáticos del Nordeste y Pampa bonaerense de Argentina. Relaciones XXXII: 101-140

CGA, Colegio de Graduados en Antropología de la República Argentina. 1989. Jornadas de Antropología: 30 Años de la carrera en Buenos Aires (1958- 1988). Mesa redonda "1983-1988" Hacia la reestructuración de la carrera: 97- 123. FFyL, UBA, Buenos Aires

CGA, Colegio de Graduados en Antropología de la República Argentina. 2011. Entrevistas realizadas por Mercedes Hirsch, Soledad Gesteira y Soledad Torres Agüero en el marco del Ciclo de encuentros "Trayectorias". http://www.cgantropologia.org.ar/trayectorias-21-luisabel-orquera

Conforti. E. y M. L. Endere. 2012. La imagen de la arqueología y el patrimonio arqueológico en los medios de comunicación. Un análisis sobre la prensa gráfica local. Antípoda. Rev. de Antropología y Arqueología, 14:163-184

Dabezies, J. M. 2011. La fotografía de campo como registro y representación: Una propuesta de análisis que explora las relaciones entre la tecnología, la técnica y el sujeto. Revista de Antropología Experimental 11: 159170.

Delfino, D. D. 2003. Entre la Arqueología y la Arquitectura, una vía de valorización sociocultural: el caso del centro de Recepción e Interpretación del Museo Integral de la Reserva de Biosfera de Laguna Blanca (Belén, Catamarca). Producciones Científicas de la Universidad de Catamarca: 14-15.

Endere, M. L. 2005. Talking about Others: Archaeologists, Indigenous Peoples and Heritage in Argentina. Public Archaeology 4: $155-162$.

Endere, M. L. y R. Curtoni. 2006. Entre lonkos y "ólogos". La participación de la comunidad indígena Rankülche de Argentina en la investigación arqueológica. Revista de Arqueología Sudamericana 2(1): 72-92.

Fernández, J. 1982. Historia de la arqueología argentina. Asociación cuyana de antropología, Talleres gráficos del Centro de Economía, Legislación y administración de Agua. Mendoza.

Fontán, M. 2005. Oswald Menghin: ciencia y nazismo. El antisemitismo como imperativo moral. Fundación Memoria del Holocausto, Biblioteca Nuestra Memoria, Buenos Aires.

Francese A., G. Migueliz, M. I. González y M. M. Frère. 2011. Proyecto de intercambio científico, artístico y pedagógico: ISFA Escuela de Cerámica De Chascomús. Provincia de Buenos Aires e Instituto de Arqueología de la Facultad de Filosofía y Letras UBA. Actas $1^{\circ}$ Congreso Docente de Experiencias Educativas Transformadoras, DGE, Buenos Aires.

Gallardo, F. A. y Cornejo, I. 1985. Definiendo el sitio arqueológico: metodología en acción. Boletín de la Sociedad Chilena de Arqueología 7: 6-11. 
Gamble C. 2002. Arqueología básica. Ariel Prehistoria, Barcelona.

González, A. R. 2000. Tiestos dispersos. Voluntad y azar en la vida de un arqueólogo. Emecé, Buenos Aires.

González de Bonaveri, M.I. y M.M. Frère. 1998. Articulación de la arqueología con los CBC en el marco de la ley Federal de Educación. Jornadas de Antropología de la Cuenca del Plata, Arqueología Tomo II: 48-55. UNR, Facultad de Humanidades y Artes. Escuela de Antropología, Rosario.

González de Bonaveri, M. I. 1997b. Technology and Mobility of Pampean Hunter Fisher Groups in Argentina. XIII UISPP Congress Procediings 5: 379-387. Forli, ABACO.

González de Bonaveri, M. I 1997a. Potsherds, "Coypo" Teeth, and Fish Bones: Hunter-Gatherer-Fishers in the Rio Salado (Pampa Region, Argentina). Quaternary of South America and Antarctic Peninsula 10: 255-278.

González de Bonveri, M. I. 1991. Tecnología de la cerámica arqueológica del Partido de Chascomús. La cadena operativa en el sitio La Guillerma 1. Arqueología 1: 105- 124.

González de Bonaveri, M. I. 1989. El uso de la imagen satelitaria como técnica de investigación arqueológica. En Primeras jornadas de becarios UBA, pp.137-139. Secretaría de Investigación y Posgrado, F. F. y L., Universidad de Buenos Aires.

González de Bonaveri, M. I. 1988. Arqueología: ¿Qué encontramos en el museo pampeano?. Informe elaborado para Museo Pampeano. Archivo Museo Pampeano, Chascomús (Inédito).

González de Bonaveri M. I. y M. A. Zárate. 1993/94. Dinámica de suelos y registro arqueológico: La Guillerma, provincia de Buenos Aires. Relaciones XIX: 285-306.

Guber, R. M. Bonnin y A. Laguens. 2007. Tejedoras, topos y partisanos. Prácticas y nociones acerca del trabajo de campo en la Arqueología y la Antropología Social en Argentina. Relaciones XXXII: 381-406

Haber et. al. 2007. Parte de la conversación. Actas del IV reunión Internacional de Teoría Arqueológica en América del Sur, Inter-congreso del WAC. pp. 61-62.

Haber, A. F. 2004. Arqueología de la naturaleza, naturaleza de la arqueología. Haber, A. Hacia una arqueología de las arqueologías sudamericanas, 15 -32, Universidad de los Andes, Facultad de Ciencias Sociales, Centro de Estudios Socioculturales e Internacionales. Bogota.
Hall, M. 2004. Romancing the stones: archaeology in popular cinema. European Journal of Archaeology 7:159 -176 .

Hodder, I. 1999. The Archaeological Process: An Introduction. Blackwell Publishers, Oxford.

Hurtado de Mendoza, D. 2010. La ciencia argentina. Un proyecto inconcluso: 1930-2000. Edhasa, Argentina.

Johnson, M. 2000. Teoría Arqueológica. Una introducción. Editorial Ariel, Barcelona.

Lahire, B. 2006. El espíritu sociológico. Manantial, Buenos Aires.

Latour, B. 1990 Drawing Things Together. Lynch M. y S. Woolgar. Representation in Scientific Practice, 19-68. MIT Press, Cambridge.

Lemaitre S. y C. Schall (comp.). 2009. How do the media represent archaeology, what is at stake?. Musees royaux d'Art et d'Histoire, Bruselas

Matera, S., M. Kergaravat, M. R. Di Donatto y F. Weber 2008. Charlas. Un encuentro con la arqueología argentina. Imago Mundi, Buenos Aires.

Mazzanti, D. 2010. Factores dominantes en el desarrollo de la arqueología pampeana del período posconquista. Nastri, J. y L. M. Ferreira. Historias de la Arqueología Sudamericana, 189-210. Fundación de Historia Natural Félix da Azara, Buenos Aires.

Mena Larraín F. 1989 Cazadores- recolectores y arqueología, Problemas y proyecciones teóricas. Boletín de antropología americana 31-47.

Nacuzzi L R. 2002 Leyendo entre líneas: una eterna duda acerca de las certezas. Visacovsky, S. y R. Guber (comps.). Historia y estilos de trabajo de campo en la Argentina, 229-262. Antropofagia, Buenos Aires.

Orquera, L. A. 1987. Advances in the archaeology of the Pampa and Patagonia. Journal of World Prehistory, 1(4):333-413.

Perez de Micou, C. 1998. Las colecciones arqueológicas y la investigación. Revista do Museu do Arqueologia e Etnología, 223-233

Podgorny, I. 2004. Tocar para creer. La arqueología en la argentina, 1910 - 1940. Anales del museo de América $12: 147-182$.

Podgorny, I. 2008. Antigüedades portátiles: transportes, ruinas y comunicaciones en la arqueología del siglo XIX, Hist. cienc. saude-Manguinhos [online] 15 no.3: 577-595. 
Disponible en http://www.scielo.br

Podgorny, I. 2009. El sendero del tiempo y de las causas accidentales. Los espacios de la prehistoria en la Argentina, 1850-1910. Protohistoria, Rosario, Argentina.

Politis G. 1992. Política nacional, arqueología y universidad en Argentina. Arqueología Latinoamericana Hoy: $70-87$.

Politis, G. y P. Madrid. 2001. Arqueología Pampeana: Estado actual y perspectivas. Berberián E. y A. Nielsen, Historia Argentina Prehispánica vol. 2, 737-814. Ed. Brujas, Buenos Aires.

Primeras Jornadas de Becarios de Investigación UBA de Filosofía y Letras. 1988. Secretaría de Investigación y Posgrado, Facultad de Filosofía y Letras, Universidad de Buenos Aires.

Pupio, M. A. 2012. Profesionales y aficionados en la conformación, interpretación y exhibición de las colecciones arqueológicas. Coleccionistas y museos de la provincia de Buenos Aires. Tesis doctoral, FFyL, UBA, Buenos Aires.

Pupio, M. A. 2005. Coleccionistas de objetos históricos, arqueológicos y de ciencias naturales en museos municipales de la provincia de Buenos Aires en la década de 1950. História, Ciências, Saúde Manghinos.Dosier Museos y Ciencias 12: 205-229.

Pupio, A. O. Heraclio I. González y M. Frere. 2004. Réplicas: manejo y conservación de objetos de alfarería. Actas del XV Congreso Nacional Arqueología Argentina, 416-417, Universidad Nacional de Río Cuarto, Río Cuarto.

Ramundo, P. 2009. Noticias en la prensa sobre arqueología argentina: una herramienta para el estudio de la historia disciplinar contemporánea. Comechingonia Virtual, III (1): 1-23.

Renfrew, C. y P. Bahn. 2011. Arqueología. Teorías, Métodos y Práctica. Akal, Madrid.

Salerno, V. M. 2011-2012. Trabajo arqueológico y representaciones del pasado prehispánico en Chascomús. Tesis doctoral, FFyL, UBA, Buenos Aires.

Salerno V. M. y A. Pupio A. 2009. Más allá de lo que se escribe: la arqueología como metáfora en los medios gráficos. Arqueología Suramericana - Arqueología sulamericana: 2(2):153-177.

Salerno, V. 2009. "Popularization" of archaeology in the Argentinian newspapers: social representation and education. Lemaitre S. y C. Schall, How do the media represent archaeology, what is at stake?, 61-71. Asbl Kinéon et ACE (Archaeology in Contemporary Europe), Proceedings of the Symposium held int the Musées royaux d'Art et d'Histoire de Bruxelles, Belgique.

Salerno, V. M. 2008. La comunicación de conocimientos arqueológicos mediante la prensa diaria en la localidad de Chascomús, provincia de Bs. As. Tesis de Licenciatura del Departamento de Ciencias Antropológicas II coordinado por Marcela Woods. Ciudad Autónoma de Buenos Aires: Editorial de la Facultad de Filosofía y Letras, Universidad de Buenos Aires. Laser Disc. Buenos Aires.

Salerno, V. y M. Vigna. 2012. Acercamiento a la construcción del pasado prehispánico en una sala del museo pampeano de Chascomús entre 1939 y 1992. Salerno, Virginia M. y Vigna, Mariana. Revista Arqueología 18, en prensa.

Thomas, D. H. 1979. Archaeology. Holt, Rinehart and Winston, New York.

Trigger, B. 1992. Historia del pensamiento arqueológico. Crítica, Barcelona.

Zárate, M. A. 1994. Geoarqueología. Jornadas de Arqueología e interdisciplinas, 21-33, CONICET, Programa de Estudios Prehistóricos, Buenos Aires.

Zárate, M. A, M. I. González de Bonaveri, N. Flegenheimer y C. Bayón. 2000/2002. Sitios arqueológicos someros: el concepto de sitio en estratigrafía y sitio de superpie. Cuadernos del Instituto Nacional de Antropología y Pensamiento Latino Americano, 19: 635- 653. 